\title{
COVID-19 vaccination associated severe immune thrombocytopenia
}

Syed Raza Ali Shah ${ }^{1,3^{*}}$, Sherpa Dolkar ${ }^{1}$, Jacob Mathew ${ }^{2}$ and Prakash Vishnu²

\begin{abstract}
Background: Coronavirus disease 2019 (COVID-19) caused by severe acute respiratory syndrome coronavirus 2 has emerged as a deadliest global pandemic after its identification in December 2019 in Wuhan, China resulting in more than three million deaths worldwide. Recently FDA issued emergency authorization for three vaccines for prevention of COVID-19. Here in, we report three cases of severe immune thrombocytopenia (ITP) following COVID-19 vaccination and their clinical course.

Case presentations: Case \#1:53 year old male with past medical history of Crohn's disease was admitted for myalgias and diffuse petechial rash 8 days after receiving second dose of Pfizer-BioNTech COVID-19 vaccine. A complete blood test showed a platelet count of $2 \times 10^{9} / \mathrm{L}$. Patient did not have a prior history of thrombocytopenia and other causes of thrombocytopenia were ruled out by history and pertinent lab data. He received two doses of intravenous immunoglobulin and oral dexamethasone for 4 days resulting in normalization of platelet counts.

Case \#2: 67 year male with past medical history of chronic ITP in remission was admitted for melena 2 days after receiving his first dose of Pfizer-BioNTech COVID-19 vaccine. A complete blood test showed a platelet count of $2 \times 10^{9} / \mathrm{L}$. Physical exam showed generalized petechiae. There was no history of recent flares of ITP and patient had normal platelet counts following his splenectomy 4 years ago. He received two doses of IVIG and oral dexamethasone for 4 days with gradual improvement in platelet counts.

Case \#3: 59 year old female with past medical history of chronic ITP secondary to SLE was admitted for bloody diarrhea 2 days after receiving her first dose of Johnson and Johnson COVID-19 vaccine. Physical exam was unremarkable. A complete blood test showed platelet count of $64 \times 10^{9} / \mathrm{L}$ which dropped to $27 \times 10^{9} / \mathrm{L}$ during hospital course. She received oral dexamethasone for 4 days with improvement in platelet counts.
\end{abstract}

Conclusion: COVID-19 vaccination induced ITP has been recently acknowledged. However, given very few cases and limited data, currently there are no guidelines for management of ITP caused by COVID-19 vaccine as well as vaccination of people with predisposing conditions.

Keywords: Immune thrombocytopenia, Coronavirus disease 2019, Pfizer-BioNTech COVID-19 vaccine, Johnson and Johnson COVID-19 vaccine

*Correspondence: syedrashah@gmail.com

${ }^{3} 4655$ Olivine Dr SW, Port Orchard, WA 98367, USA

Full list of author information is available at the end of the article

\begin{abstract}
Introduction
Immune thrombocytopenia (ITP) is an acquired hemorrhagic diathesis characterized by a platelet count of $<100 \times 10^{9} / \mathrm{L}$ caused by immune-mediated destruction of platelets, impaired production or increased splenic sequestration [1]. ITP is idiopathic in $80 \%$ of cases, and primary ITP is often thought to be an autoimmune
\end{abstract} permits use, sharing, adaptation, distribution and reproduction in any medium or format, as long as you give appropriate credit to the original author(s) and the source, provide a link to the Creative Commons licence, and indicate if changes were made. The images or other third party material in this article are included in the article's Creative Commons licence, unless indicated otherwise in a credit line to the material. If material is not included in the article's Creative Commons licence and your intended use is not permitted by statutory regulation or exceeds the permitted use, you will need to obtain permission directly from the copyright holder. To view a copy of this licence, visit http://creativecommons.org/licenses/by/4.0/. The Creative Commons Public Domain Dedication waiver (http://creativeco mmons.org/publicdomain/zero/1.0/) applies to the data made available in this article, unless otherwise stated in a credit line to the data. 
condition. However, $20 \%$ of cases of ITP are usually secondary to an underlying precipitating etiology such as infection, medications, rheumatologic disorders or malignancy. Occurrence of ITP has also been reported following vaccinations against various infectious agents especially measles-mumps-rubella (MMR), but also Hemophilus influenza, hepatitis B virus, human papilloma virus, varicella-zoster, polio and pneumococcus [2]. Recently, FDA issued emergency use authorization for three vaccines for the prevention of coronavirus disease 2019 (COVID-19) caused by severe acute respiratory syndrome coronavirus 2 (SARS-CoV-2) [3]. Few cases of ITP after SARS-CoV-2 vaccination with both the Pfizer and Moderna vaccines have been reported and have reached public attention. Herein, we report three cases of severe ITP that occurred following COVID-19 vaccination and their outcome.

\section{Case presentations Case \#1}

A 53-year-old man with past medical history of Crohn's disease well-controlled with Ustekinumab, and no prior history of thrombocytopenia or exposure to heparin was seen in Emergency Department at St. Michael Medical Center Silverdale, Washington (ED) with symptoms of intermittent episodes of high-grade fever with chills, diffuse myalgia and petechial rash over chest, abdomen and extremities that had started 2 days prior. He did not have symptoms of coryza, cough, headache, abdominal pain, rectal bleeding, dysuria, diarrhea or altered sensorium. He had received the second of the two doses of PfizerBioNTech COVID-19 vaccine 8 days prior to the onset of symptoms. In the ED, he had a temperature of $39^{\circ} \mathrm{C}$. He had petechiae in the oropharynx, anterior trunk and bilateral upper and lower extremities. Rest of the physical exam was unremarkable. A complete blood count (CBC) showed a platelet count of $2 \times 10^{9} / \mathrm{L}$. Rest of the blood counts and red cell indices were normal. Peripheral blood film did not show platelet clumping or schistocytes. Current list of medications didn't reveal any culprit medications and he had normal platelet count $\left(254 \times 10^{9} / \mathrm{L}\right)$ 5 weeks prior. Urinalysis, Blood cultures and X-ray chest did not show any evidence of infectious etiology and he remained afebrile throughout hospital course. Viral respiratory panel including influenza $\mathrm{A}$ and $\mathrm{B}, \mathrm{RSV}$ and COVID-19 was negative. Hepatitis C and HIV serology was non-reactive. Coagulation profile was normal. Computed tomography (CT) of the head, chest, abdomen and pelvis did not show any evidence of thrombosis. Spleen measured $14.2 \mathrm{~cm}$. Complement levels and autoimmune panel including anti double stranded DNA antibody and anti CCP antibody was normal. Bone marrow biopsy showed normocellular bone marrow with mildly increased megakaryopoiesis, preserved trilineage hematopoiesis without atypia or increased blasts. Flow cytometry didn't show any abnormal B cells without an increase in CD 34 positive blasts. He received intravenous immunoglobulin (IVIG) $1 \mathrm{~g} / \mathrm{kg} /$ day for 2 consecutive days and oral dexamethasone $40 \mathrm{mg} /$ day for 4 consecutive days. With this regimen, the platelet count normalized in about 4 days after initiation of therapy and the response was durable. (Fig. 1).

\section{Case \#2}

A 67-year-old man with past medical history of seizure disorder, atrial fibrillation, chronic ITP in remission and no prior exposure to heparin, was seen in ED with symptoms of generalized weakness and melena. Patient reported that he had received the first dose of PfizerBioNTech COVID-19 vaccine two days prior to admission. Patient denied any recent flares of ITP and he had normal platelet counts in the range of $140-160 \times 10^{9} / \mathrm{L}$ since undergoing splenectomy 4 years ago. Physical exam showed generalized petechiae. Rectal exam revealed black tarry stools. Rest of the physical exam was unremarkable. A CBC showed a platelet count of $2 \times 10^{9} / \mathrm{L}$. Rest of the blood counts and red cell indices were normal. Peripheral blood film did not show platelet clumping or schistocytes. Hepatitis C and HIV serology was non-reactive. Viral respiratory panel including influenza $\mathrm{A}$ and B, RSV and COVID-19 was negative. Coagulation profile was normal. Patient received two units of single-donor platelet transfusion without any significant improvement in platelet count, however melena stopped. CT of the head and chest were unremarkable while the $\mathrm{CT}$ of the abdomen showed mild rectal wall thickening with fat stranding suspicious of proctitis. The patient received IVIG $1 \mathrm{~g} / \mathrm{kg} /$ day for 2 consecutive days and oral dexamethasone $40 \mathrm{mg}$ /day for 4 consecutive days. He had a gradual improvement in platelet count which eventually normalized in about a week after initiation of therapy. (Figure 2) Patient was advised to defer the second dose of COVID-19 vaccine due to the occurrence of severe thrombocytopenia.

\section{Case \#3}

A 59-year-old woman with past medical history of chronic ITP and systemic lupus erythematosus (SLE) on hydroxychloroquine was seen in ED for symptoms of abdominal cramps and diarrhea that started 2 days after she had received Johnson and Johnson Covid-19 vaccine. Patient's previous flare of ITP was two years ago which was precipitated by Shingrex vaccine that was treated successfully with steroids, following which her platelet count remained stable $\left(\sim 100 \times 10^{9} / \mathrm{L}\right)$. Physical exam was unremarkable. A complete blood 




Fig. 1 Platelet count before, during and after hospitalization for ITP with timeline of relevant events

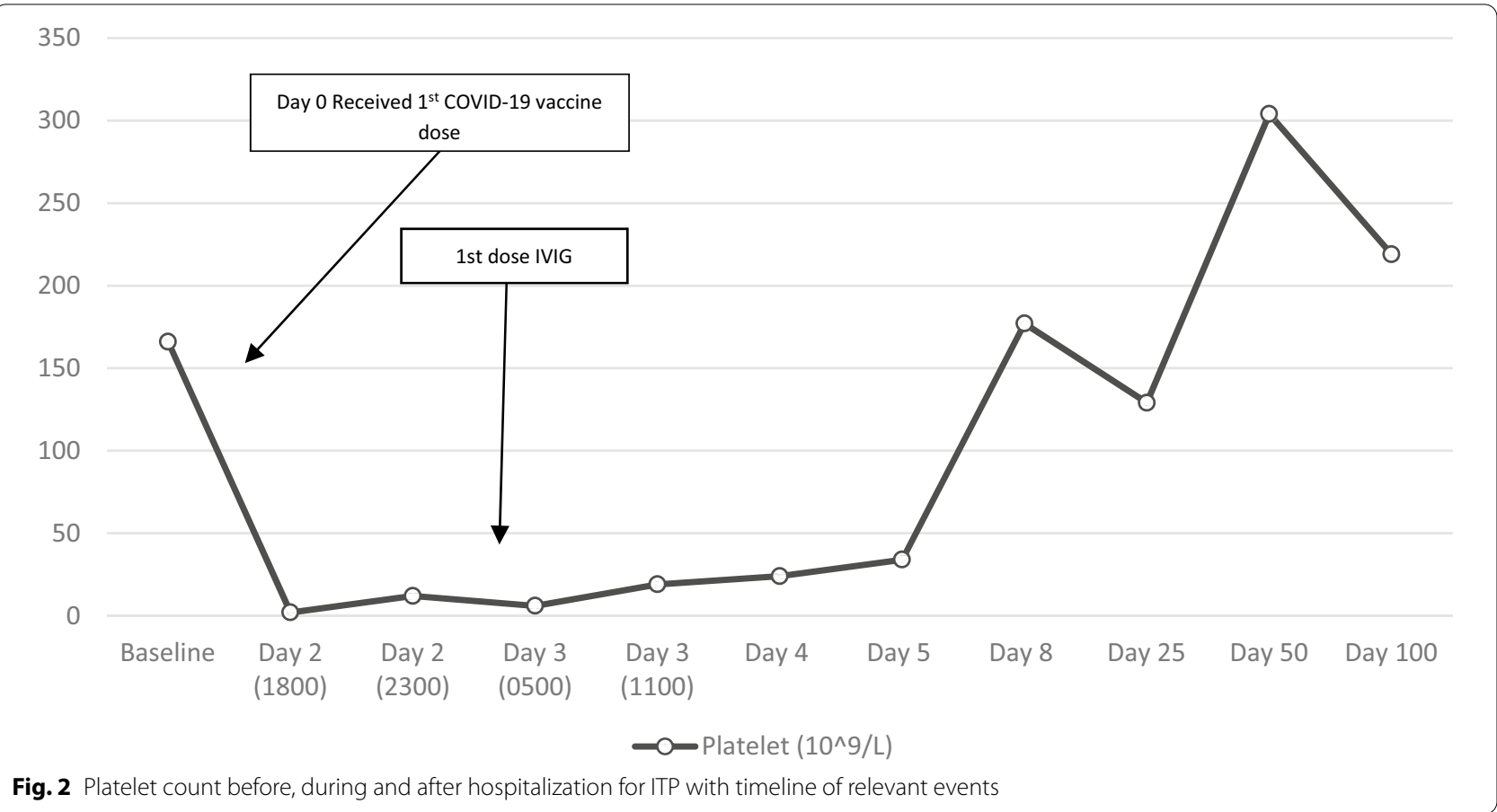

test showed a platelet count of $64 \times 10^{9} / \mathrm{L}$ and subsequently dropping to $27 \times 10^{9} / \mathrm{L}$ the following day. Peripheral blood film did not show platelet clumping or schistocytes. CT abdomen showed mild distal colitis. Prior laboratory data showed non-reactive
HIV and hepatitis B and C serology. Patient received oral dexamethasone $40 \mathrm{mg}$ /day for 4 days with gradual improvement in platelet count as shown in Fig. 3. 


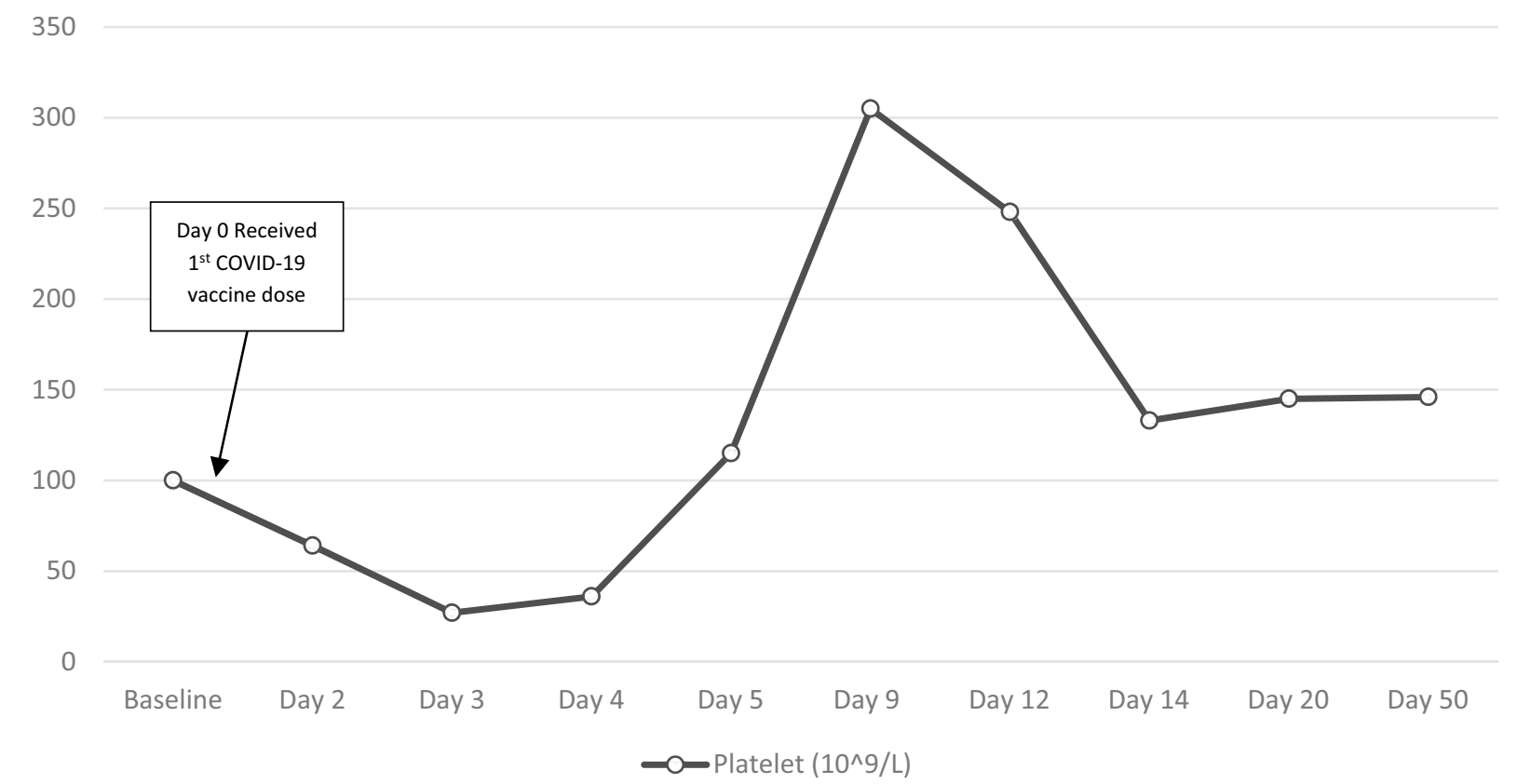

Fig. 3 Platelet count before, during and after hospitalization for ITP with timeline of relevant events

\section{Discussion}

Vaccination associated ITP is a rare phenomenon. ITP after COVID19 vaccination is relatively new occurrence, and the management and outcomes of these cases are not well published in medical literature. Only recently, a case series reported twenty patients with thrombocytopenia following vaccination, based on the data obtained from the Centers for Disease Control and Prevention (CDC), FDA and agencies of the U.S. Department of Health and Human Services (HHS) Vaccine Adverse Events Reporting System (VAERS) [4]. Also, an anecdotal case of a 56-year-old physician who had developed severe thrombocytopenia after receiving COVID-19 vaccine and eventually died due to hemorrhagic stroke was reported in The New York Times [5].

Our report describes three cases of severe ITP that occurred following COVID-19 vaccination and also provides insights into the treatment and outcomes for ITP secondary to COVID-19 vaccination. All three patients responded well to treatment with corticosteroids \pm IVIG. If the treatments (IVIG and/or steroids) utilized in our cases were not effective, and the platelet count remained very low, it would then be reasonable to escalate therapy to include a thrombopoietic agent, and potentially vinca alkaloids, depending upon response. Avoiding rituximab from initial treatment would be appropriate since the response to rituximab may take up to 6-8 weeks; furthermore, the response to COVID-19 vaccine may be impaired by rituximab. Whether post COVID-19 vaccination ITP cases will prove to be self-limiting or persist and lead to chronic ITP remains uncertain. It is also unclear if the ITP is secondary to the vaccination or primary ITP that was coincident with the vaccine. Nevertheless, the clinical manifestation and the positive response to treatment akin to ITP, such as with corticosteroids \pm IVIG, suggest an antibody-mediated platelet destruction.

ITP has been previously reported with different types of vaccines including hepatitis B virus (HBV) human papilloma virus (HPV) varicella zoster, haemophilus influenza, polio, pneumococcus, Diphtheria-tetanus-acellularpertussis (DTap) and especially measles-mumps rubella (MMR) [6]. Proposed mechanisms include molecular mimicry and antibody generation to adjuvants/additives included in the vaccine composition which can lead to generation of autoantibodies that cause platelet destruction and inhibit platelet production [7]. Data also show that post-vaccination ITP ranges in severity from mild to moderate to severe and is highly responsive to IVIG treatment as seen in our patients [8]. Literature review shows conflicting data as some studies show evidence of ITP reactivation when exposed to the same vaccine while others do not demonstrate the ITP reactivation when the vaccine is repeated [9-12]. Many authors believe ITP is not an absolute contraindication for vaccination given a much higher rate of ITP linked with natural infection as compared to vaccination however recommend to avoid vaccination during active ITP flare [13]. 


\section{Conclusion}

As mass vaccination is in progress, more data should be collected to establish a cause-effect relationship of COVID-19 vaccination and associated ITP so that the CDC can formulate guidelines for safe administration for COVID-19 vaccination. This can be done by outlining possible contraindications and potential treatment measures for ITP so as to prevent fatal outcomes. Additionally, more research may be needed to make changes in vaccine composition to prevent thrombocytopenia. One consideration could be to monitor patients with history of ITP for an antibody response and reserving the second dose of the vaccine for those without an adequate response. Until there is further development of appropriate guidelines, physicians should discuss these risks and benefits.

\section{Abbreviations}

ITP: Immune thrombocytopenia; COVID-19: Coronavirus disease 2019; SARSCoV-2: Severe acute respiratory syndrome coronavirus 2; ED: Emergency Department; CBC: Complete blood count; CT: Computed tomography; IVIG: Intravenous immunoglobulin; SLE: Systemic lupus erythematosus; CDC: Centers for Disease Control and Prevention; FDA: U. S. Food and Drug Administration; HHS: Department of Health and Human Services; VAERS: Vaccine adverse events reporting system; HBV: Hepatitis B virus; HPV: Human papilloma virus; DTap: Diphtheria-tetanus-acellular-pertussis; MMR: Measles-mumps rubella.

\section{Acknowledgements}

Not applicable.

\section{Authors' contributions}

Conception of the work: SRAS and PV. Manuscript preparation: SRAS and PV. Data acquisition: SRAS and SD. Manuscript review and final approval: SRAS, SD, JM and PV. All authors read and approved the final manuscript.

\section{Funding}

The authors declare that no funding was received for this study.

\section{Availability of data and materials}

The data used during the current study is available from the corresponding author on reasonable request.

\section{Declarations}

Ethics approval and consent to participate

Saint Michael Medical Center Ethics Committee approved the study.

\section{Consent for publication}

Informed consent was taken from all three patients.

\section{Competing interests}

The authors declare that they have no competing interests.

\section{Author details}

${ }^{1}$ Departments of Hospital Medicine, St. Michael Medical Center, Virginia Mason Franciscan Health, Silverdale, WA, USA. ${ }^{2}$ Departments of Hematology, St.

Michael Medical Center, Virginia Mason Franciscan Health, Silverdale, WA, USA.

${ }^{3} 4655$ Olivine Dr SW, Port Orchard, WA 98367, USA.

Received: 1 June 2021 Accepted: 7 July 2021

Published online: 15 July 2021

\section{References}

1. Rodeghiero F, et al. Standardization of terminology, definitions and outcome criteria in immune thrombocytopenic purpura of adults and children: report from an international working group. Blood. 2009;113(11):2386-93.

2. David P, Shoenfeld Y. ITP following vaccination. Int J Infect Dis. 2020;99:243-4.

3. https://www.fda.gov/news-events/press-announcements/fda-issuesemergency-use-authorization-third-covid-19-vaccine. Accessed 27 Feb 2021

4. Lee E-J, et al. Thrombocytopenia following Pfizer and Moderna SARSCoV-2 vaccination. Am J Hematol. 2021;96(5):534-7.

5. https://www.usatoday.com/story/news/health/2021/01/06/death-flori da-doctor-following-pfizer-covid-19-vaccine-under-investigation-grego ry-michael/6574414002/. Accessed 06 Jan 2021.

6. Perricone C, et al. Immune thrombocytopenic purpura (ITP) associated with vaccinations: a review of reported cases. Immunol Res. 2014;60(2-3):226-35

7. Watad A, et al. Autoimmune/inflammatory syndrome induced by adjuvants (Shoenfeld's syndrome) —an update. Lupus. 2017;26(7):675-81.

8. Rajantie J, et al. Vaccination associated thrombocytopenic purpura in children. Vaccine. 2007;25(10):1838-40.

9. Beeler J, et al. Thrombocytopenia after immunization with measles vaccines: review of the vaccine adverse events reporting system (1990 to 1994). Pediatr Infect Dis J. 1996;15(1):88-90.

10. Bibby AC, et al. Is MMR immunization safe in chronic Idiopathic thrombocytopenic purpura? Arch Dis Child. 2008;93(4):354-5.

11 Hamiel U, et al. Recurrent immune thrombocytopenia after influenza vaccination: a case report. Pediatrics. 2016;138(6): e20160124.

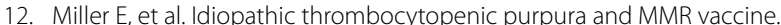
Arch Dis Child. 2001;84(3):227-9.

13. Cecinati $\vee$, et al. Vaccine administration and the development of immune thrombocytopenic purpura in children. Hum Vaccines Immunother. 2013;9(5):1158-62.

\section{Publisher's Note}

Springer Nature remains neutral with regard to jurisdictional claims in published maps and institutional affiliations.

\footnotetext{
Ready to submit your research? Choose BMC and benefit from:

- fast, convenient online submission

- thorough peer review by experienced researchers in your field

- rapid publication on acceptance

- support for research data, including large and complex data types

- gold Open Access which fosters wider collaboration and increased citations

- maximum visibility for your research: over 100M website views per year
}

At BMC, research is always in progress.

Learn more biomedcentral.com/submissions 